\title{
mHealth for transgender and gender-expansive youth: harnessing gender-affirmative cross-disciplinary innovations to advance HIV prevention and care interventions
}

\author{
Simone J. Skeen ${ }^{1}$, Demetria Cain ${ }^{1}$, Kristi E. Gamarel ${ }^{2}$, Lisa Hightow-Weidman ${ }^{3}$, Cathy J. Reback ${ }^{4,5}$ \\ ${ }^{1}$ PRIDE Health Research Consortium, Hunter College of the City University of New York, New York, NY, USA; ${ }^{2}$ Department of Health Behavior \\ \& Health Education, University of Michigan School of Public Health, Ann Arbor, MI, USA; ${ }^{3}$ Institute for Global Health and Infectious Diseases, \\ The University of North Carolina at Chapel Hill, Chapel Hill, NC, USA; ${ }^{4}$ Friends Research Institute, Inc., Los Angeles, CA, USA; ${ }^{5}$ Department of \\ Family Medicine, Center for HIV Identification, Prevention and Treatment Services, University of California Los Angeles, Los Angeles, CA, USA \\ Contributions: (I) Conception and design: All authors; (II) Administrative support: All authors; (III) Provision of study materials or patients: None; (IV) \\ Collection and assembly of data: SJ Skeen; (V) Data analysis and interpretation: SJ Skeen; (VI) Manuscript writing: All authors; (VII) Final approval \\ of manuscript: All authors. \\ Correspondence to: Simone J. Skeen. PRIDE Health Research Consortium, Hunter College of the City University of New York, New York, NY, USA. \\ Email: sskeen@prideresearch.org.
}

\begin{abstract}
Transgender and gender-expansive (TGE) youth endure stark disparities in health and wellbeing compared to their cisgender peers. A key social determinant of health for TGE adolescents and emerging adults is gender affirmation, which encompasses multidimensional validations of an individual's lived gender. Lacking available resources for one's gender affirmation, TGE young people may engage in highrisk maladaptive coping behaviors, linked to their disproportionately high HIV-acquisition risk. A range of innovative mobile technologies are guided by the Gender-Affirmative Framework to promote the health of TGE communities, including through HIV prevention and care continuum outcomes. The aim of this review was to examine key features of existing mobile technologies that can be leveraged to advance the field of TGE-responsive mHealth. We systematically searched scientific records, gray literature, and the iOS and Android app distribution services. To be eligible, platforms and interventions needed to be tailored exclusively to a TGE user base, incorporate gender-affirming features, and be optimized for or adaptive to mobile technologies. Eligible interventions $(\mathrm{N}=24)$ were compared on evidence of utility, core functionalities, and dimensions of gender affirmation. Smartphone applications (apps) and webapps ( $\mathrm{n}=16)$ were the most common delivery modality. Many interventions $(n=9)$ aimed to address HIV-related outcomes and integrated gender-affirmative features. The most common gender-affirmative features originated in fields of human-computer interactions and informatics, or were crowdfunded by TGE developers. HIV-focused interventions incorporated evidence-based health behavior change strategies and utilized rigorous evaluation methods. Across modalities and disciplines, behavioral self-monitoring and access to HIV prevention services were the most frequent features. Over two-thirds of the interventions reviewed aimed to provide medical gender affirmation (e.g, provided guidance on obtaining medically sanctioned hormone therapies, or safely practicing non-medical options such as chest-binding) or psychological gender affirmation (e.g, provided linkage to mental health counseling). Our results show that mHealth and other technology-mediated interventions offer a diverse range of both evidence-based and innovative features; however, many have not been rigorously evaluated in a randomized controlled trial to support TGE users. A continuing commitment to evidence-based health behavior change strategies, exemplified by the HIV-focused interventions included in this review, is essential to advancing gender-affirmative mHealth. The unique and highly innovative features of platforms originating outside the fields of HIV prevention and care suggest new directions for TGE-responsive mHealth, and the need for more conscientious models of knowledge exchange with investigators across scientific disciplines, private-sector developers, and potential users.
\end{abstract}

(C) mHealth. All rights reserved. 
Keywords: Gender affirmation; HIV; human-computer interactions; mHealth; technology; transgender; youth

Received: 07 February 2020; Accepted: 03 June 2020; Published: 20 April 2021.

doi: $10.21037 /$ mhealth-20-60

View this article at: http://dx.doi.org/10.21037/mhealth-20-60

\section{Introduction}

Despite some advances in public awareness and legal protections, transgender and gender-expansive (TGE) youth continue to face grave health inequities that stem from multiple sources, such as biased and exclusionary laws and policies, societal discrimination, and a lack of access to quality and affirming health care (1), increasing their risk for HIV acquisition (2): among transgender people living with HIV in the U.S, 36.3\% were youth under the age of 24 (3). The field of HIV prevention is increasingly moving towards technology-mediated interventions for youth, as mobile health (mHealth) and electronic health (eHealth) are easily accessible and can be delivered in real time (4). However, despite the promise of technology-mediated interventions, there has been limited focus on how these interventions can be harnessed to address HIV prevention and care for TGE youth $(5,6)$.

This review was specifically focused on youth who fall under the umbrella of TGE, including those who may be actively questioning their gender $(7,8)$. To clarify our discussion, we begin by providing some key definitions; however, these brief descriptions may not capture all of the nuances in this rapidly evolving field. "Sex", or "natal gender", is a label, which is generally defined as "male" or "female", that is assigned at birth, while, "gender identity" is one's inherent sense of self, which results from a multifaceted interaction of biological traits, developmental factors, and societal context (9). "Gender expression" refers to the different ways people display their gender through clothing, hair styles, mannerisms, or social roles (10).

These labels may or may not be congruent. The term "cisgender" is used when someone identifies with and expresses a gender that is consistent with the culturally defined norms of the sex that was assigned to them at birth (11). "Transgender" is usually reserved for the subset of individuals whose internal gender identity is inconsistent with the sex assigned on their birth certificate (12). These terms are not diagnoses but are instead personal and often dynamic ways of describing one's gender experience. "Gender diverse" or "gender expansive" are umbrella terms that individuals may apply when their gender identity, expression, or physical attributes do not conform to the norms and stereotypes others expect of their assigned sex (13). More recently, the terms "nonbinary" or "genderqueer" have been adopted within scientific discourse and among many TGE communities to refer to individuals who do not conform to binary conceptualizations of gender as either male or female (14). In this review, we intentionally use the terms $T G E$ to describe all non-cisgender youth, as some people use multiple gender identities and, additionally, some gender identities change across one's life course. For example, some nonbinary people identify as transgender; however, others do not (8). Thus, the term gender-expansive provides a means to begin to accommodate different experiences, expressions, and selfidentifications (8).

Although few probability studies assess gender identity, it's been estimated that $0.7 \%$ to $3.2 \%$ of youth identify as transgender $(15,16)$. These adolescents and emerging adults can find social inclusion elusive, and may lack access to tailored health resources. A recent nationally representative survey of 5,765 TGE teens, ages 13-17, found that less than a quarter feel safe being themselves at home and in school; $72 \%$ have heard their families make denigrating remarks toward sexual and gender minorities; and $84 \%$ have been threatened at school, a setting in which only $10 \%$ reported receiving safer sex information responsive to their lives and identities (8). TGE youth, particularly youth of color, are also at substantial risk of a multitude of adverse outcomes, with $25-43 \%$ experiencing unstable housing/homelessness $(17,18), 66 \%$ enduring unemployment $(18,19), 67 \%$ engaging in survival sex (17), and 1 in 10 surviving physical assault (20). To date, the majority of research among TGE youth has focused on the role stigma and discrimination play in creating or exacerbating these adverse social and health conditions (21).

More recently, the field has moved towards embracing gender affirmation as a key social determinant of health for gender-expansive people $(22,23)$. The "Gender Affirmation Framework" refers to a multifaceted and often longterm process through which TGE individuals align their internal senses of embodiment and external expressions of 
culturally bound gender norms with their inherent gender identities (24). Not getting gender affirmation needs met across different dimensions such as medical (e.g, exogenous hormone therapy to develop a more gender-congruent sense of embodiment, including through physical features), social (i.e, use of correct name and pronouns), psychological (i.e, perceptions of respect), and legal (i.e, name change documentation) can place TGE youth at increased risk of emotional distress, suicidality, sexual risk behaviors, medical mistrust $(23,25,26)$, and serve as a barrier to engaging in HIV prevention and care (27). According to the Gender Affirmation Framework (24), lacking accessible sources of gender affirmation, TGE individuals may seek validation of their emerging identities through high-risk behaviors such as condomless anal sex (CAS) and bodily modifications in non-medical settings. TGE youth have reported rates of CAS between $27-48 \%$ and non-medical injection of hormones or silicone between 24-27\% (28). Widespread stigma, sexism, and other vectors of oppression place already marginalized subpopulations, such as TGE youth of color, at particularly high risk of HIV acquisition (24). Given the complex risk profiles of many TGE communities, innovative approaches that address both gender affirmation and HIV prevention and care are needed.

\section{Technology engagement among TGE youtb}

Teenagers across the LGBTQ spectrum report spending an average of 5 hours online every day, 45 minutes longer than their non-LGBTQ peers, with $76 \%$ preferring online chat or text message options when seeking support in a crisis $(29,30)$. Within the LGBTQ spectrum, 26\% of transgender youth communicate online with LGBTQ friends daily or almost daily, while $20 \%$ or less of their cisgender peers exhibit similar frequency. Using the internet to acquire health information was near-universal among transgender youth: $95 \%$ report doing so, versus $80 \%$ or less of their LGB peers. In regards to accessing HIV/AIDS and STI information online, transgender youth were outpaced only by cisgender gay and bisexual young men (29). Given these patterns of technology use $(4,5,31)$, technology-mediated modalities are ideal for delivering gender-affirming health interventions to TGE young people, including interventions focused on HIV prevention and care. Social media, and other online venues that enable peer-to-peer interaction, selective self-presentation, and the sharing of user-generated content $(32,33)$, can be essential sources of gender affirmation for TGE youth (34-36).
The features of these platforms can subvert the isolation and routine invalidations endured by TGE youth who do not have access to affirming homes or schools. For example, TGE Tumblr users can obtain social connection by making meaning of their experiences, establishing peer-to-peer "care structures" navigable via an array of gender-specific hashtags $(37,38)$. TGE youth and their caregivers can equip themselves with clarifying terminologies and discover both on- and offline support networks through listservs, social forums, and the websites of local nonprofits (39). But the unique features of online platforms also enable harms that disproportionately impact TGE communities. Cyberbullying, harassment, stalking, and other abusive online behaviors are directed toward TGE youth at disparate rates (40). Many, as a result, may feel anxious, depressed, or suicidal $(29,41,42)$. Misinformation and non-evidence-based anecdotes on gender-affirming care discoverable online can jeopardize TGE young people's wellbeing (39).

For many TGE youth, technology-mediated interventions are situated within this rich, yet fractious, online milieu, in which TGE youth spend approximately 5 hours daily (29). Recent mHealth interventions, which aim to build upon evidence-based bio-behavioral HIV prevention strategies, intend to activate this online milieu to advance the health and wellbeing of these vulnerable communities (43). Notably, the field of HIV prevention is not alone in advancing genderaffirming mobile interventions for TGE communities. The importance of gender affirmation in promoting the health and wellbeing of TGE communities has driven the development of other, distinct lineages of technologymediated interventions tailored toward the needs of TGE communities $(44,45)$. Often, these platforms are aligned with human-computer interaction (HCI) disciplines, or rely on crowdfunding, open-source architectures, and robust community engagement for sustainment (46-48). The core functionalities of these platforms intend to aid TGE users in affirming their gender identities and navigating their lives safely as TGE individuals $(45,49)$. Notably, the functionalities often do not explicitly engage with traditional HIV prevention and care strategies; however, their emphases on gender affirmation is aligned with contemporary understandings of HIV acquisition among high-risk TGE populations. Specifically, these functionalities frame HIV transmission risk behaviors as maladaptive attempts to attain gender affirmation in oppressive, invalidating settings, where safer sources of 
Table 1 Conceptualization of key terms in research question

\begin{tabular}{|c|c|c|}
\hline Key term & Definition & Source \\
\hline Young TGE communities & $\begin{array}{l}\text { Adolescents and emerging adults whose gender transcends } \\
\text { the sex assigned to them at birth (i.e., transgender) including } \\
\text { individuals who do not embrace the "transgender" label (i.e., } \\
\text { gender-expansive) }\end{array}$ & $\begin{array}{l}\text { Gender-Expansive Youth Report, HRC: } \\
\text { Human Rights Campaign, 2018:5 (8) }\end{array}$ \\
\hline
\end{tabular}

validation are unavailable (24).

As such, a cross-disciplinary scoping review of smartphone-based, SMS, telemedicine, and other TGEfocused mobile technology-mediated interventions has the potential to provide new directions in honing the next generation of mHealth designed to improve HIV prevention and care with and for TGE youth. The present review aims to identify and describe a diverse catalog of technological platforms guided by the tenets of the Gender Affirmation Framework. In doing so, we adopt the lens of Affordance Theory, which views technological features in terms of imagined affordances: the human-perceptible actions and possibilities inherent in new technologies, emphasizing the previously inconceivable expectations and socio-cultural meanings they may accrue over time. "Human-perceptible", in this context, refers to possibilities that the end-user is aware of and, therefore, may integrate into their daily lives (50). Thus, a focus on affordances foregrounds new potentials that rely on the unique interplays between human and technological agency (51).

\section{Methods}

We used the Arksey and O'Malley five-stage framework for scoping review studies $(52,53)$. A scoping review was chosen over a systematic review so that we could include a broad range of technology-mediated interventions, use a multi-pronged search strategy to capture traditional clinical trials (in progress, where necessary), and communityderived innovations across disciplines. We did not place any restrictions on publication type. In this, our approach is consistent with recent scoping reviews in mHealth and eHealth (54-57).

\section{Stage 1: identifying the research question}

The rationale for the review arose from the discontinuity and lack of knowledge sharing in the areas of genderaffirmative technology-mediated interventions evident across disciplines-specifically, between HIV-focused mHealth and parallel developments in informatics, HCI, and outside of traditional research infrastructures entirely. Our objective was to analyze these rich but varied advances through a single lens, guided by the Gender Affirmation Framework, and the abovementioned concept of imagined affordances.

Thus, the research question guiding our scoping review was: "How has customized software, optimized for or adaptive to mobile technologies (including $m$ Health), aimed to advance the bealth and wellbeing of young TGE communities by affirming their lived genders?"

The conceptualization of our key terms, in emulation of the format presented by Adepoju et al. (58), are shown in Table 1. 
Table 2 Inclusion and exclusion criteria applied

\begin{tabular}{lll}
\hline Aspect & Included & Excluded \\
\hline $\begin{array}{l}\text { Primary intended } \\
\text { users }\end{array}$ & $\begin{array}{l}\text { Platforms and mobile/telehealth interventions tailored } \\
\text { for TGE individuals and communities exclusively, } \\
\text { of adolescent, emerging adult, or unspecified age } \\
\text { range-but not explicitly tailored for older adults }\end{array}$ & $\begin{array}{l}\text { Platforms for undefined/universal user bases and clinicians } \\
\text { exclusively (e.g., e-Consult); those tailored for broader } \\
\text { sexually and gender-diverse communities, which may or may } \\
\text { not include TGE individuals; and those tailored for cisgender } \\
\text { users seeking sexual encounters with TGE individuals }\end{array}$ \\
& $\begin{array}{l}\text { Platforms that aim to advance aspects of medical/ } \\
\text { embodied, psychological, social, and/or legal gender } \\
\text { affirmation, or to advance circumstances through } \\
\text { which gender diversity can be safely and openly lived }\end{array}$ & $\begin{array}{l}\text { Platforms that lack interactivity (e.g., static websites, net } \\
\text { art), consist solely of games or passive media, or consist } \\
\text { exclusively of geosocial/sexual networking or dating features }\end{array}$ \\
& $\begin{array}{l}\text { Platforms optimized for or adaptive to mobile } \\
\text { networked technologies such as smartphones, } \\
\text { tablets, and smartwatches }\end{array}$ & $\begin{array}{l}\text { Platforms exclusive to desktop computers or other non- } \\
\text { mobile technologies, or telehealth extensions of primarily } \\
\text { brick-and-mortar providers }\end{array}$
\end{tabular}

Telehealth interventions were compiled prior to the COVID-19 pandemic, which has led to an immense scaling up of telehealth deployments in response to widespread physical distancing measures $(66,67)$.

\section{Stage 2: identifying relevant studies}

To capture empirical articles, formative research, and other traditional publication types in medical, behavioral, and socio-technical science, PubMed, PsycINFO, and the ACM (Association for Computing Machinery) Digital Library were queried. To capture gray literature such as project reports, funding prospectuses, blogs, and popular media reporting, Google and DuckDuckGo (a search engine on the open web that forgoes the algorithmically personalized rankings that can render Google's search results impossible to replicate) (63) were used. Recent reference lists were hand-searched by the first author (SJS). All records were compiled and screened using the Zotero reference manager (64).

Search terms were developed iteratively, based on pilot searches in Google Scholar. Our final terms comprised "trans*gender", "genderqueer", "non*binary", "gender expansive", "gender diverse", "agender", "youth", "adolescent", "online”, "web app”, “tech*”, "counsel*”, "HIV", "health", "mobile", and "text message" alone and in combination. Results were unrestricted by date.

Additionally, Google Play and the App Store, the mobile app distribution services for the Android and iOS smartphone operating systems, respectively, were searched directly for software relevant to our abovementioned objective. Each was queried with the term "transgender", and the full results were screened against our selection criteria.

Our search was limited to English-language reporting and platforms. All searches were undertaken initially by the first author (SJS) in August, 2019, with secondary title/ abstract screening by a research intern (AP) in November,
2019. All databases were systematically re-queried, and our results updated, in April and May, 2020.

Finally, as the authors are involved in various roles within the UNC/Emory Center for Innovative Technology (iTech) (65), we were able to include the integrated TechStep platforms prior to any external reporting on that intervention.

\section{Stage 3: study selection}

The inclusion of mHealth and other platforms in this review was dependent on the selection criteria shown in Table 2. Out of our initial 565 candidate platforms identified via title/abstract screening, gray literature searches, app distribution services, and the iTech network, 24 were selected for inclusion. The complete process is illustrated in Figure 1.

\section{Stage 4: charting the data}

Consistent with Arksey \& O’Malley (52), we developed a descriptive-analytical summary of our findings, delineating key aspects of each platform for comparison, with a focus on distinguishing novel gender-affirming functionality. The respective platforms' key features were categorized inductively by the first author with the aid of a consensus coder (AP).

The four recognized dimensions of gender affirmation were adapted from the typology of gender affirmation as a social determinant of health described by Reisner et al. (23), 

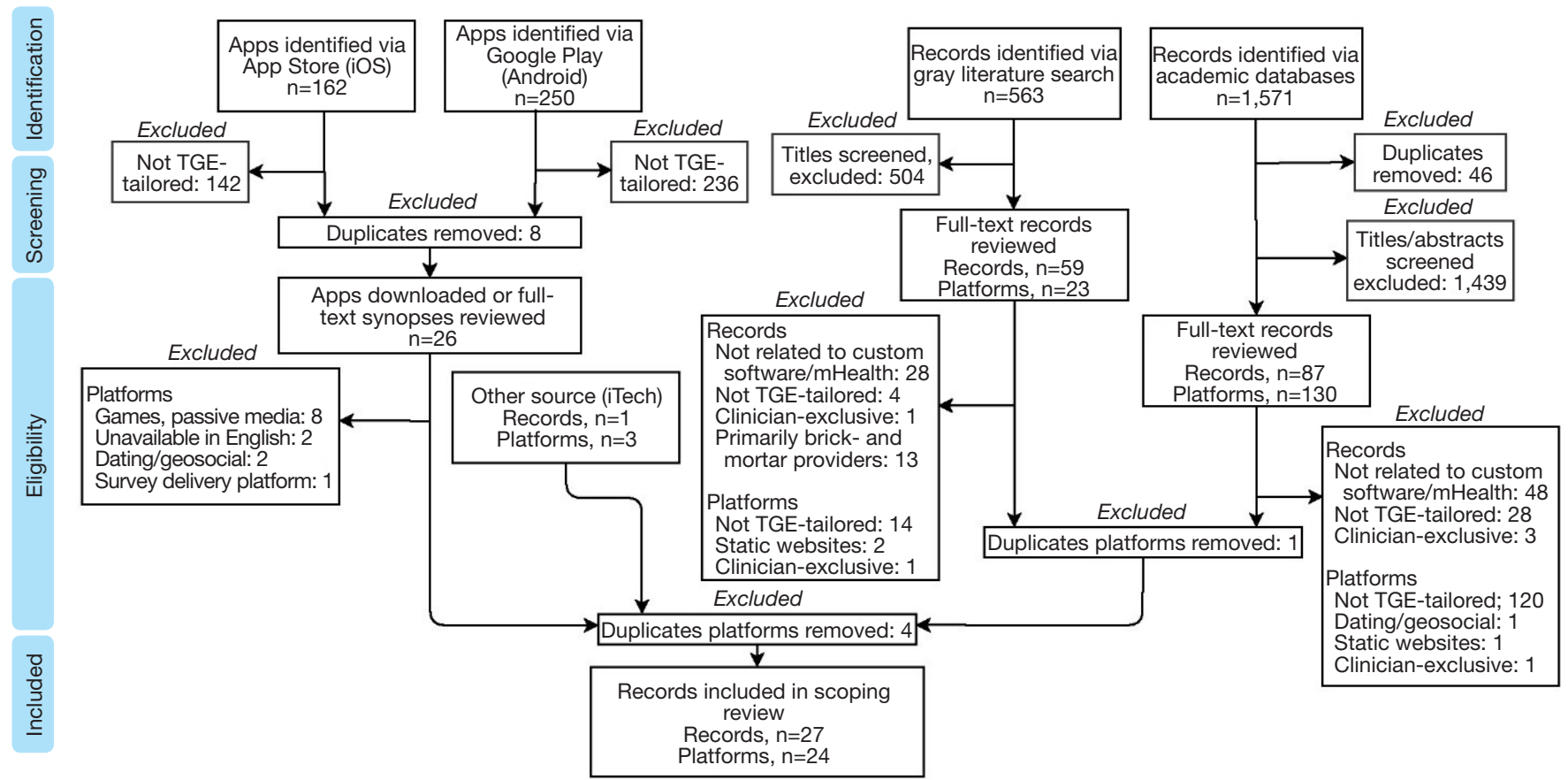

Figure 1 Flow diagram illustrating the search and selection of commercially available and in-development or mid-evaluation platforms and interventions.

and defined in the previous section. Because many approaches to bodily gender affirmation (e.g, chest binders, electrolysis) can be accessed outside of medical settings, we replace "medical" with embodied in the present typology. These dimensions were assigned by the first author with the aid of a second consensus coder (PG). In both coding procedures, discrepancies were resolved in conference until unanimity was reached.

\section{Stage 5: collating, summarizing, and reporting the results}

We then compiled and summarized the results of each of the platforms and report on comparisons between platforms in the use of evaluation methods, theoretical grounding, key/unique features, and dimensions of gender affirmation.

\section{Results}

The final sample included 24 platforms or interventions, in widely disparate phases of development. Their names and brief descriptions, modes of delivery, state of their evidence bases, theoretical groundings (if specified), key or noteworthy features, and the dimensions of gender affirmation they aim to engage, are presented in Table 3.

\section{Outcomes and efficacy}

Of the 24 platforms examined, nine included HIV prevention and treatment outcomes, with seven platforms HIV focused; three had undergone pilot studies that provided acceptability and feasibility outcomes, and only one used a fully powered randomized controlled trial (RCT) to evaluate intervention efficacy. One pilot study, Project Moxie, which is a remote video counseling intervention, found that approximately half of the total participants assigned to the intervention group took part in the counseling sessions; however, the TGE participants who did take part reported uniformly high rates of satisfaction with the counseling and software. Although sexual risk behaviors remained unchanged at 3 months, there was a significant increase in STI testing rates and PrEP acceptability among participants at 3 months (73). Trans Women Connected, a small acceptability and usability pilot study, showed increases in self-efficacy to access specialized and online support (102). Similarly, the unnamed Washington, DCbased telehealth intervention, which included HIV services among its referrals, showed increases in users' intentions to seek TGE-affirming and other specialized care (104). Text $M e$, Girl!', which provided trans women with supportive text 
Table 3 Summary of technology-mediated ( $N=24$ ) interventions tailored for or inclusive of TGE youth

\begin{abstract}
description
Binder Reminder is tailored toward trans-masculine, nonbinary, and other users who wear compressive chest binders to reduce the appearance of natal breasts. The app consists of binder or undertake brief stretching exercises. These features aim to stem the discomfort and shortness of breath that can accompany uninterrupted binding (68). The developer engages the community on Tumblr, where updates are shared and user feedback is solicited (46).

Christella VoiceUp: voice modification toolkit (69), cf. EVA: Exceptional Voice App.
\end{abstract}

EVA: Exceptional Voice App comprises a suite of tools, in "MTF" and "FTM" editions. Developed by a speech pathologist, EVA offers users educational modules and biofeedback binary, cis-centric, norms (49).

PatchDay: hormone self-monitoring platform (72) cf. Trans Memo.

Project Moxie combines online HIV test counseling with self-administered rapid HIV esting via kits mailed to participants. The intervention, which deploys HIPAA-secure VSee videoconference software, combines $\mathrm{Ml}$ and counseling, testing, and referral approaches to address HIV transmission risk and testing among TGE youth, with linkage to medical care and other services, as indicated (6).

QueerDoc: online-only gender-affirming telemedicine practice (75), cf. QMed.

Smartphone app:

IV prevention-

sed

E

Evidence summary

No Binder Reminder formal outcomes evaluations have been reported.

Explicit theoretical grounding

Key/unique features

Behavioral se

monitoring

Enbodion

Embodied;
psychological;

social

No

\section{Smartphone app:}

Cf. EVA: Exceptional Voice App.

n/a

EVA and interventions with equivalent functionality [e.g., Christella VoiceUp (69)] $\mathrm{n} / \mathrm{a}$

have not been subjected to formal outcomes evaluation. But formative research in

$\mathrm{HCl}$ has interrogated the pathologizing and rigidly binary assumptions ingrained

in these apps' features, calling their acceptability into question (70). Contrary to

the assimilationist framing of these apps' primary outcomes, most trans-efemining
participants ( $N=7, \mathrm{M}_{\mathrm{age}}=26.3$ ) recounting their experiences with voice training

were motivated by physical safety concerns and self-affirmation (71).

Smartphone app: No

OS exclusive

Telemedicine/

No PatchDay formal outcomes evaluations have been reported.

$\mathrm{n} / \mathrm{a}$

Project Moxie recruited 202 participants, ages 15-24, who did not identify with

the sex assigned to them on their birth certificate, to take part in a pilot study.

While approximately half of participants assigned to the intervention took part in

the counseling sessions, satisfaction with the counselors and the videoconference
software, and willingness to repeat and recommend the intervention, were both

uniformly high among this cohort. Willingness to use PrEP saw increases in both

the intervention and control samples. Gains in STI testing were shown at 3 mon

in the intervention sample (73).

Telemedicine/ online video

counseling

No, but PrEP and Cf. QMed. STI testing are

QMed [aka QueerMed] is a telemedicine practice that offers specialized trans-affirmative healthcare to TGE youth across the southeastern U.S., with a focus on serving adolescents in online video rural settings. Deploying HIPAA-secure videoconference software, TGE and gender-questioning counseling young people can be evaluated for hormone therapy, and connected to labs for bloodwork and mental health counseling, as indicated (76).

QueerViBE is a private web-based platform that provides trans-masculine and nonbinary youth - Internet/webapp with 6 interactive YouTube tutorials, of 6-12 minutes, which aim to subvert hegemonic cis-

centric noms of masculinity and hus to validate the identities and expressions of its users. Its sexual and gender-minority male youth ( $\mathrm{n}=28$, ages $16-24)$.

Refuge Restrooms maps publicly accessible single-occupancy and gender-neutral restroon for TGE and intersex users who may feel uncomfortable or be targets of harassment and

violence in binary gendered toliet facilities (47). Listings on Refuge Restrooms can be

submitted, rated, and commented upon by users. It is open source, and offers features closely

equivalent to tis now-noperative forebears (cc. GotrourBack (84); Transquat (85)) and ple
of equivalent functionality (cf. Safe Transgender Bathroom App (88). As well, the Refuge

Restrooms database is now available as a contact on the $Y O$ messaging app allowing users

to send a "YO" to the database and, in response, receive a hyperlocal listing of safe restroom

based on their present location (87).

$$
\text { Table } 3 \text { (continued) }
$$

๑ mHealth. All rights reserved.
QMed and practices with equivalent models of care [cf. QueerDoc (75)], have not been subjected to formal outcomes evaluation. But their feasibility for rural TGE patients may be hampered by unreliable Internet connectivity, a state-bystate patchwork of medical licensure among practitioners, and inconsistent telemedicine parity laws, which require insurers to compensate providers at rates equivalent to an in-office visit, by state (77).

In a preliminary RCT, which recruited trans-masculine, nonbinary, genderquestioning, and intersex participants ( $N=156$, ages 15-21), QueerViBE showed significant improvements in the trial's primary outcome variables: At 1-month follow-up, individual and collective self-esteem, wellbeing, and positive tran identity were elevated among QueerVIBE users compared to a wailist cont
CBT (74); hegemonic

masculinities (78); heterosexua matrix (79); invalidation and microaggressions (80); policing masculinities (81); power and
Behavioral selmonitoring:

biofeedback

Behavioral self-

monitoring:

Embodied; psychological; socia Embodied; psychological;

Embodied;
psychologica Behavioral se

monitoring

HIV testing and Psychological

counseling; remote

video counseling

Remote video

counseling

Embodied,

Remote video

counseling

Embodied; legal

Psychological; 
Table 3 (continued)

Name, description

Safe Transgender B
Refuge Restrooms.

ShotTraX: hormone self-monitoring platform (88), cf. Trans Memo.

Solace LGBT, a smartphone app, presents users with a customizable menu of common gender-transition goals, e.g., updating legal documents, acquiring new wardrobes, and accessing hormone replacement therapies with necessary multidisciplinary assessments and diagnostic prerequisites by U.S. state or territory. The app includes a progress tracker (cf. Trans Tracks (89), for equivalent functionality) that encourages users to monitor their progress toward selected goals; a passcode system to protect their privacy; integration with Respite, an aggregator of news relevant to TGE

Spokane Trans Map is a smartphone app focused on an interactive map of Spokane, Washington, allowing TGE contributors to signify TGE-friendly businesses with green mark

and TGE-hostile businesses with red markers, based on their personal experiences. Singleoccupancy restrooms are mapped as well A project of the Solce LGBT developer, Spokane

TechStep: webapp provides users a customizable profile; a "wall" on which to post personal updates, photos, videos, and to respond to other users' posts; multimedia content curated by TGE youth; resources for HIV testing, PrEP, PEP, hormone therapy, housing, legal, and abuse support services, and a customizable behavior tracker, which prompts users to administer

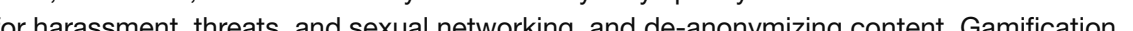
elements allow users to unlock new customization options with routine use of TechStep's features. These features reflect the insights of TGE youth focus groups, convened prior to the webapp's development (43). Resources are currently limited to the trial's subject recruitment venue cities.

TechStep: SMS presents participants with 3 text messages daily for 90 days, grounded in Social Support Theory (social support), Social Cognitive Theory (self-efficacy), and the Health Belief Model (health-protective behaviors and health threats). Each theory corresponds to 90 distinct messages along the HIV Prevention Continuum, for a total library of 270 messages. The ext messages cover holistic understanding of

TechStep: eCoaching is an online counseling intervention tailored toward TechStep: webapp Telemedicine/online Yes and TechStep: SMS users who could benefit from a more intensive model of care. At the trial's 3-month assessment, TechStep users engaging in refractory sexual risk behaviors may be re-randomized to the "eCoaching" intervention. Its $5-8$ sessions, which rely on HIPAAsecure Zoom videoconference software and novel integrated plattorms for both eCoach and communiction, self-reguldion self-monitoring, and problem-soling skils, tallod to build needs and experiences of high-risk TGE youth (43).

Modality

\section{HIV prevention-
or care-focused}

Evidence summary

Evidence summary

the

\author{
he
}

Explicit theoretical grounding

reported.

n/a

No ShotTrax formal outcomes evaluations have been reported.

No Solace LGBT formal outcomes evaluations have been reported.
No Spokane Trans Map formal outcomes evaluations have been reported.

The TechStep 3-arm RCT study, which includes the webapp, SMS, and eCoach components (see below), is actively enrolling HIV-negative participants that are at risk of HIV acquisition and who do not identify with their assigned sex (target $\mathrm{N}=250$, ages 15 -24). Primary outcome variables include reduced sexual risk post-enrollment, compared to a static TGE-tailored and HIV/STI- informative website. Secondary outcomes include experienced transphobia, housing precarity and other structural factors as moderators of sexual risk (43).

Immediate and sustained effects will be compared to those of the TechStep: webapp (see above) condition on the RCT's primary outcomes of interest (43).

video counseling 
Table 3 (continued)

Name, description

Text Me, Girl! aims to improve health outcomes among young adult HIV-seropositive trans women. Specifically, recipients were sent 3 text messages daily (from a library of 270) within a customizable timeframe. Similar to TechStep: SMS (see above), but for young adult trans women living with HIV, the Text Me, Gir!! message content was theoretically grounded in Socid Support Theory (social support), Social Cognitive Theory (self-efficacy), and the Health Belfor Model (health-proteclive behaviors and heall hireats). Comm unlly insights informed the (con

they2ze combines regularly updated multimedia curated by TGE youth; resources for employment services, food, emergency financial assistance, online peer forums, hormones, outbound links to evidence-based information on gender-affirming surgeries, and fertility and mmigration concerns for TGE communities. Educational modules for providers, aiding then in building a trans-affirmative practice, are included. they2ze incorporates the insights of a community advisory board of TGE youth. Currently, resources are limited to the San Francisco Bay Area (98).

Trans Memo [aka Testo Memo] provides TGE users a simple interface to set reminders to administer their hormones. It includes options to monitor supplies and track the regularity 列 equivalent to similar plattorms such as PatchDay (72) and ShotTraX (88).

Trans Pelvic Health [aka Kegel Nation] is a prototype that enables users recovering from gender-affirming genital reconstructive surgeries to track pelvic floor exercises, urinary

frequency and urgency, and regularity of douching and dilating the neovagina, as indicated. Is features are adaptable to surgical procedures typically undertaken by both trans-masculne and trans-feminine individuals (100)

TransTracks: gender transition progress tracker (89), cf. Solace LGBT (48).

$\begin{array}{ll}\text { Modality } & \text { HIV prevention- } \\ \text { or care-focused }\end{array}$
or care-focused

Evidence summary

Explicit theoretical groundin

Text Me, Girl! enrolled 130 young adult (ages 18-34) trans women living with HIV. At Health belief model (92); sociobaseline, only $35 \%$ of participants were virally suppressed, and only $5 \%$ reported cognitive theory $(93,94)$ their ART medication adherence as "excellent." By 18 -month distal follow-up,
of the participants were virally suppressed and $38 \%$ had achieved "excellent"

of the participants were virally suppressed and $38 \%$ had achieved "excellent"
ART adherence. ART uptake also significantly increased from $49 \%$ at baseline to

ART adherence. ART uptake also significantly increased from $49 \%$ at baseline to
$77 \%$ at 18 -month distal follow-up. Multivariate analyses indicated that increased $77 \%$ at 18 -month distal follow-up. Muttivariate analyses indicated that increased of attending a HIV doctor visit in the past six months, and increased probability of achieving an undetectable viral load. Multivariable results also indicated that retention in Text Me, Girl! was associated with significantly improved ART adherence, and significantly increased likelihood of achieving an undetectable vira oad (97).

Smartphone app: Yes iOS and Android

Yes

No they2ze formal outcomes evaluations have been reported

A small usability and acceptability study ( $N=10$, ages $40-70$ ) recorded high ease population (100)

Trans Women Connected is an mHealth app undergoing iterative prototyping at the time of writing. Its functionality is grounded in qualitative formative research with trans-feminine communities, and its developers, UX specialists, and engineers worked in tandem with an eported prototype incorporates a vision board permitting users to set personal goals across structural domains such as schooling, employment, and housing (as well as motivations such as "making a difference"), allowing its trans-feminine target users to collage their own photos and/or create original images and text; educational video modules on PrEP, and a map of loca PrEP providers, and an interactive resource map that taps users' smartphone GPS to show

Table 3 (continued)

\section{Smartphone app: Yes}

Smartphone app:
iOS and Android

Yes

Unspecified

Formative work comprised a series of 4 focus groups and 20 individual intervieus the importance of structural determinants of trans women's health, their holistic understandings of their sexual health, and desires for supportive bonds with other trans women (101). These insights informed the current prototype, which underwen usability and acceptability evaluations with trans-female testers $(\mathrm{N}=16)$. Ratings overall were high, with significant post-est increases in self-efficacy to access GGBTQ+ services, intention to seek online support, and PrEP knowledge. Following furher prototyping, including plans to embrace elements of CBT and the Gender cluster RCT (102).
Key/unique features

TGE-curated

Embodied; legal:

mortar resources; HIV

Provider education

Embodied

Behavioral self-

monitoring

Embodied;

Gender affirmation

HIV testing and counseling; TGEresources; TGEcurated brick-andmortar resources; Usergenerated content 
Table 3 (continued)

Name, description

Modality

Evidence summary
Explicit theoretical grounding
Key/unique features

Gender affirmation

(Unamed telehealth intervention, Washington DC]. Deploying the HIPAA-secure Digigone videochat app, alongside texting, emailing, and telephone options, this telehealth intervento
provided Washington, DC-based trans women of color with 24-hour access to local peer

health consultants, a model that has shown success in serving other populations with uniq

Telemedicine/online No, but HIV care was The intervention was grounded in insights provided by trans women of color and

healthcare needs (103). The aim of the intervention was to leverage telehealth and mobile

technology toward overcoming the structural barriers faced by many trans women of color
when they try to secure healthcare access. The peer consultants provided "one-stop shopping

when they try to secure healtcare access. The peer consullants provided one-stop shopp

programs - in response to queries by users (103), with monthly consultant-initiated check-

ins in the absence of any other contacts. Physicians were available at all times to address

emergencies or urgent issues beyond the purview of the consultants.

U-Signal is a prototype that combines smartphone and smartwatch functionalities to support Smartphone, the safety of trans-feminine and nombinary users. By tapping the smartwatch screen. U-Signal smartwatch

can be subtly activated, sending an SMS or voice-recorded alert to a user's social network,

and communicating their GPS coordinates consistently thereafter. Customizable user profiles

eir needs $(\mathrm{N}=22$, including 4 trans-female

providers) in the Washington, $\mathrm{DC}$, area. The qualltative data focused on housing

stability as a key determinant of health and wellbeing, the primacy of hormone

access among healthcare needs, and pervasive experiences of discrimination,

stigma, and medical mistrust. In turn, the peer-dellvered telehealth model was

unwelcoming helthcar settings (103). In a small ( $\mathrm{N}=25$ ) feasibity study enrolling

trans women of color with experiences of encountering structural barriers to care

in the prior 6 months, the 3-month intervention was linked to significant increases

in intention to seek TGE-responsive care, and intentions to seek specialty, mental health, and HIV care among users with depressive symptoms (104).

The app's features were based on 9 initial interviews and 7 follow-up interviews

with trans women, and trans-feminine and nonbinary people of color. The

feedback suggests high acceptability with the bypassing of police and medics in

favor of peer networks portrayed as "everyone's favorite" feature. Concerns around

expense and accessibility, particularly for non-English speakers, were raised (45).
HIV testing and Embodied; legal;

counseling; remote psychological;

curated informative

resources

Customizable user
profiles; Geolocatio

tracking and

networking; Personal

safety; socia

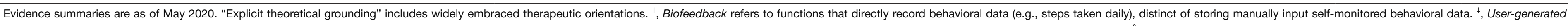

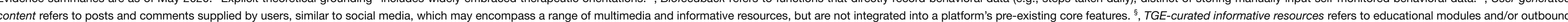

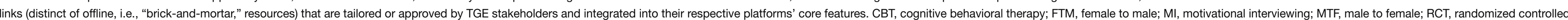
trial; TGE, transgender and gender-expansive. 
messages, demonstrated significant outcomes for increased antiretroviral treatment (ART) uptake, excellent ART adherence, and viral suppression among trans-feminine young adults (ages 18-34) living with HIV (97). As of May 2020, TechStep, with its webapp, SMS, and eCoaching platforms (which we reviewed independently) are in the midst of a randomized controlled trial to determine their efficacy in reducing behavioral risk for HIV/STI acquisition and encouraging PrEP uptake and adherence (43). The they2ze app, developed by the nonprofit YTH [Youth + Tech + Health (98)], and the QueerDoc telehealth service (75), both include linkages to HIV services. However, as of this writing, no data regarding intervention outcomes or efficacy have been reported for either platform.

The other platforms $(n=15)$ whose primary aims did not focus explicitly on HIV prevention or care have not presented evaluation data to establish their efficacy or effectiveness. Many appeared to be early in development and pilot testing; thus, qualitative feasibility and acceptability data provided by small groups of target users was most commonly provided. For instance, U-Signal, an integrated smartphone-smartwatch platform designed to enable peer-to-peer alerts of imminent personal safety risks among trans-feminine and nonbinary communities of color, showed strong acceptability among seven TGE interviewees who reviewed the prototype (45). Trans Pelvic Health, a prototype iOS app that tracks key aspects of self-administered aftercare for gender-affirming genital surgeries (e.g, vaginoplasty), such as urinary frequency and routine Kegel exercises, show satisfactory preliminary usability among 10 pilot users, though it is unclear if these users were TGE (100). The exception was QueerViBE, which comprises a series of online educational modules, grounded in discursive analysis of TGE youth focus groups, and aiming to evoke self-esteem and positive transgender identity among its trans-masculine and nonbinary users, QueerViBE demonstrated preliminary efficacy using an RCT design; however, the study lacked follow-up measures beyond one month (44).

\section{Theoretical grounding}

Among the 24 platforms, six described their theoretical groundings, which were mostly focused on $\operatorname{HIV}(n=5)$, with the exception of QueerViBE. The two SMS-enabled interventions reviewed, TechStep: SMS and Text Me, Girl!, were grounded in the Health Belief Model, Social Cognitive Theory, and Social Support Theory, aiming to identify health-protective behaviors and health threats, bolster individuals' self-efficacy, and embrace available instrumental, emotional, and informational supports, respectively $(43,97)$. The TechStep: webapp intervention was developed based on the Information Motivation Behavioral Skills (IMB) model, which highlights the role of informational attainment and motivational drivers in the adoption of health-protective behaviors (91). Motivational interviewing (MI) guided both Project Moxie and TechStep: eCoaching; the latter of which employed a hybrid MICognitive Behavioral Therapy (CBT) approach to empower TGE users through evoking their own capacities for selfdetermination and health-protective decision-making $(6,43)$.

The only intervention not explicitly addressing HIV outcomes that provided evidence of a theoretical basis for development was QueerViBE, which, in line with its aims to enhance a multilevel framework of wellbeing, ranging from the individual to socio-political, integrates multiple social constructionist and queer theories. These include analyses on the social construction of masculinity (the platform was tailored for trans-masculine and nonbinary youth), its societally enforced norms (81), and their relations to visibility and power, interpreted through a Foucauldian lens $(82,83)$. In addition, one module also incorporates CBT techniques, aiming to preserve users' self-esteem when confronted with intrusive and invalidating questions from cisgender peers (44).

\section{Dimensions of gender affirmation}

All but one of interventions profiled $(n=23)$ sought to address psychological dimensions of gender affirmation, by providing linkage to mental health counseling or by attempting to boost user self-esteem and/or self-efficacy. Fifteen interventions had features and resources aiding in social affirmation, which included, for example, features connecting TGE peers and mentors, and activating community networks and crowdsourced knowledge. Embodied affirmation was addressed in another 15 interventions through guidance on accessing hormones and safely undertaking non-medical bodily affirmation options. In more than half the interventions reviewed $(n=15)$, psychological and social affirmation co-occurred, typically through core features designed to enhance both social supports and agentic, individual, wellbeing. These were evident in the webapps with social networking features (TecbStep: webapp, Trans Women Connected, U-Signal) and the SMS-enabled interventions (TecbStep: SMS and Text Me, 
Girl!). The embodied-psychological $(\mathrm{n}=13)$ and embodiedsocial $(n=6)$ dimensions were less likely to co-occur. They appeared typically — though not exclusively-in the platforms that incorporate a broad range of resources for medical, mental health counseling, and community support referrals. Only six provided legal gender affirmation, typically through referrals to legal assistance options, with the exception of QMed, which provides required medical documents for legal gender-marker and name updates directly. Only Solace LGBT, TechStep: webapp, Trans Women Connected, and the Washington DC intervention engaged in all four dimensions recognized by the Reisner et al. (23) gender affirmation typology.

\section{Key and unique features}

The most frequently integrated features were behavioral selfmonitoring ( $\mathrm{n}=11)$, compilations of TGE-responsive brickand-mortar resources $(\mathrm{n}=8)$, and informative content tailored by TGE stakeholders ( $\mathrm{n}=7)$. TechStep: webapp and they2ze, both focused on HIV prevention and sexual health education among TGE users, were the most feature rich. HIV testing and counseling resources and remote video counseling were available almost exclusively within the HIV-prevention interventions, with the exception of the QueerDoc and Washington, DC-based telehealth interventions. Only Refuge Restrooms, Safe Transgender Batbroom App, the Spokane Trans $M a p$, and $U$-Signal incorporate features designed to enhance personal safety: Refuge Restrooms (and similar platforms; see Table 3), through mapping all-gender and single-occupancy publicly accessible restrooms for users, who may fear harassment or violence in traditionally gendered spaces (47); and U-Signal, through its unique peer-to-peer alerts and the discretion with which they can be activated, silently, by smartwatch (45).

\section{Discussion}

TGE young people endure compounded vulnerabilities to stigma, social isolation, violence, and HIV (9). While the adoption of digitally networked technologies among TGE youth is widespread, their online social milieu can be both robustly supportive and a site of invalidation and abuse (42). A diverse array of mobile technologies aims to promote TGE users' health and wellbeing, primarily through gender-affirmative and HIV-prevention features. These interventions incorporate both traditional mHealth elements, such as behavioral tracking and hyperlocal referrals, and highly innovative platform-specific features, tailored for a TGE user base. This review examined these interventions by evaluation methods, dimension of gender affirmation, feature profiles, and their respective evidence bases.

The most evident distinction between disciplines is the commitment to randomized controlled trials of efficacy, which was all but unique to the HIV-prevention interventions. These platforms, through government funding and accountability structures, and the involvement of university-affiliated investigators, require "gold-standard" evidence of their efficacy and effectiveness via longitudinal and resource-intensive trials (105). Translationally, this distinction is paramount, as future funding, widespread dissemination, and the resources for adaptation rely on established efficacy. For example, Project Moxie, TechStep (across modalities), Text Me, Girl!, and Trans Women Connected are supported by research infrastructures able to rigorously assess feasibility, acceptability, and efficacy outcomes (65). The app and webapp interventions focused on HIV prevention and care also possessed the most features among the interventions reviewed. Additionally, they2ze, TechStep, and Trans Women Connected, all integrate a spectrum of high-quality curated resources conscientiously tailored for their target TGE users.

On the contrary, the HIV-prevention interventions lack the unique, core affordances that have guided the conspicuously leaner non-HIV-focused platforms. Refuge Restrooms and Safe Transgender Bathroom App, for example, perform a single function (locate restrooms). But through this single function, they reliably create new possibilities for personal safety among their users. This leanness, even if it sacrifices functional heterogeneity, enables access for lower socioeconomic status and transient users, who may share devices, use lower-specification hardware, or be subject to intermittent connectivity and power outages (106). The features exclusive to the non-HIV-focused platforms focus on within-person drivers of gender affirmation. Some of these platforms are narrow in focus [Trans Memo, Binder Reminder $(46,99)]$, whereas others seek to holistically address gender affirmation needs [EVA, Solace, TransTracks $(48,49,89)]$, Others acknowledge the often-politicized meanings that TGE communities may attach to-or have imposed upon-their identities [QueerViBE, U-Signal $(44,45)]$. Together, the non-HIV-focused platforms included in this review illustrate the importance of routine selfvalidation and physical safety as critical needs for TGE youth. Thus, while low-threshold hormone access is a key 
component of TGE wellbeing (as QMed recognizes), it is distinct, experientially, from the routinely accessible and cumulative validations these platforms aim to provide. While TechStep: webapp and other platforms offering peerto-peer interactivity are potentially sources of routine social support, the unpredictable nature of user-generated content is unlikely to achieve the consistent benefits conveyed by such finely honed features as QueerViBE's discursively grounded educational modules.

Similarly, while tips for physical safety may, or may not, be exchanged among socially networked peers, freedom from harassment and violence was the sole aim of Refuge Restrooms and U-Signal (45,47). Physical safety concerns often drive the uptake of $E V A$ and other voicetraining interventions (71). Mistrust of law enforcement is highly prevalent among TGE communities, and in particular among Black and Latinx trans communities (107), accounting for the peer-to-peer alerts enabled by $U$-Signal. Just as QueerViBE's educational modules acknowledge the lived reality of stigmatization among its users, and their possible internalization of power asymmetries in a cissexist society, U-Signal acknowledges the mutually compounding systems of oppression of racism, sexism, and anti-transgender stigma for the trans-feminine users of color for whom it was designed. The HCI-derived, crowdfunded, and other non-HIV-focused interventions, in short, conceive their features in terms of resistance to the structural subordinations that drive health inequities among TGE communities. Interpreted through an imagined affordance lens, these features-and the expectations and new potentials they permit TGE users-can be understood as tools to ease the daily burden of these subordinations.

These new possibilities for self-validation, safety, and adaptive identity formation, highlight the promise of such innovative features to communities of users who share complex, distinct, and often overlapping needs. The concept of affordances, within psychology, referred originally to the instrumental possibilities of an environment, as recognized by a specific individual or group within it (50). Maintaining an imagined affordance-focused lens can aid in honing the aims of innovative features, and clarifying the insights provided by TGE communities throughout the evaluative cycle (e.g, formative, process). In short, what are the expectations and new potentials that TGE users desire of mobile, networked technologies? How does the provision of these new potentials address the health inequities endured by TGE youth, including their disparate risk of HIV acquisition? The realization of new potentials within the digitally networked environment by TGE developers, investigators, and communities, should serve to inspire the future of government-backed and university-affiliated mHealth interventions tailored to meet their needs.

\section{Advancing the field}

The landscape of TGE-responsive mobile technology, mapped in this review, points to a number of promising directions.

\section{Knowledge exchange}

Future practice and research could focus on understanding how to balance and bridge fields that often operate in siloes. The innovations advanced by TGE private-sector developers and TGE-focused investigators in HCI and social informatics suggests that a cross-sectoral stakeholder engagement or knowledge exchange model of intervention development could elicit promising new insights, while ensuring proper crediting of community-derived innovations. One such example is the mHealth intervention TreatYoSelf. While not TGE-focused, it is exemplary of such an approach, leveraging the patient-clinician-designer framework of participatory design, and allying experts in HCI with clinician-investigators in adolescent HIV (108). In capturing imagined affordances, the openly utopian "future-making" workshop process can record both the structural barriers encountered by communities attempting to realize their potential—and activate their imaginations toward surmounting those barriers with highly speculative original technologies (109).

\section{Theoretical synthesis}

The creatively realized affordances shown by the nonHIV-focused platforms are not inherently at odds with the theoretical underpinnings of much HIV-focused mHealth. Peer-to-peer empowerment, behavioral self-monitoring, and tailored informational sources are embraced across disciplines, and their potential benefits could be catalyzed by a more thorough and thoughtful grounding in health behavior change theories.

\section{Functional integration}

With the HIV-focused platforms often feature rich, and the non-HIV-focused platforms often lean but highly innovative, opportunities for high-impact functional integration may present themselves. Some instances of such convergent functionality, aligning HIV- and non-HIV- 
focused developments, are evident already: TechStep: webapp, for example, mimics the full functionality of Trans Memo, via its customizable behavioral trackers. But with accessibility for at-risk and under-resourced users tied to leanness [versus "feature creep" (110)], calibrating the balance versus overproliferation of features will remain an important goal.

\section{Outcomes evaluation}

Multi-center longitudinal RCTs are often not accessible, nor appropriate, for all platforms. However, evaluations of desired outcomes, such as pre- and post-test designs, and explorations of acceptability, usability, and sustainable sociocultural attunement (e.g, the incorporation of fast-evolving linguistic norms across TGE youth communities) can build a case for functional integration with feature-rich, evidencebased, mHealth interventions.

\section{Limitations}

There are several noteworthy limitations. The timeframe within which this review was conducted limited the state of the evidence we were able to present. With findings of acceptability, feasibility, and ultimately efficacy more fully reported, the foundations for the recommendations offered here may shift substantially. Similarly, we were unable to capture the abrupt scaling up of telemedicine opportunities, including for TGE patients, which has occurred in response to the COVID-19 pandemic of $2020(66,67)$. Furthermore, time and resource constraints prevented us from undertaking the optional expert consultation exercises recommended by Arksey and O'Malley (52,53). Given the nascent and discontinuous advances we aimed to map in this review, such an exercise would be especially conducive to bridging knowledge bases. As such, our results should be understood as a selective and vision-setting overview of an evolving field, rather than an integrative review of methodology or efficacy.

\section{Conclusions}

TGE adolescents and emerging adults endure disproportionate rates of HIV-acquisition risk, violence, and emotional distress. While networked and mobile technologies carry risks of their own, their outsized adoption by TGE youth points toward promising mHealth modalities. By aligning the micro-targeted socio-cultural attunements of community-backed interventions with the robust scientific infrastructures of government-funded HIV prevention and care programming, strides in acceptability, feasibility, and lasting utility can be achieved.

\section{Acknowledgments}

The authors would like to thank AvaGrace Palazzolo and Patrick George III for their aid in database searching and retrieval, and developing and triangulating the codes in Table 1. The authors also thank Dr. Sonia Lee for her support of this work.

Funding: This research was supported by a National Institutes of Health grant (U19HD089881). The content is solely the responsibility of the authors and does not represent the official views of the funding agencies. KEG acknowledges additional support from the National Institute of Mental Health (R25MH067127) and Eunice Kennedy Shriver National Institute of Child Health and Human Development (L30HD098047). CJR acknowledges additional support from the National Institute of Mental Health (P30MH58107).

\section{Footnote}

Provenance and Peer Review: This article was commissioned by the Guest Editor (Lisa Hightow-Weidman) for the series "Technology-based Interventions in HIV Prevention and Care Continuum among American Youth" published in $m$ Health. The article has undergone external peer review.

Conflicts of Interest: All authors have completed the ICMJE uniform disclosure form (available at http://dx.doi. org/10.21037/mhealth-20-60). The series "Technologybased Interventions in HIV Prevention and Care Continuum among American Youth" was commissioned by the editorial office without any funding or sponsorship. LHW served as the unpaid Guest Editor of the series. Dr. Hightow-Weidman reports grants from NICHD, during the conduct of the study. The authors have no other conflicts of interest to declare.

Ethical Statement: The authors are accountable for all aspects of the work in ensuring that questions related to the accuracy or integrity of any part of the work are appropriately investigated and resolved.

Open Access Statement: This is an Open Access article distributed in accordance with the Creative Commons Attribution-NonCommercial-NoDerivs 4.0 International 
License (CC BY-NC-ND 4.0), which permits the noncommercial replication and distribution of the article with the strict proviso that no changes or edits are made and the original work is properly cited (including links to both the formal publication through the relevant DOI and the license). See: https://creativecommons.org/licenses/by-nc-nd/4.0/.

\section{References}

1. Gridley SJ, Crouch JM, Evans Y, et al. Youth and caregiver perspectives on barriers to gender-affirming health care for transgender youth. J Adolesc Health 2016;59:254-61.

2. Becasen JS, Denard CL, Mullins MM, et al. Estimating the prevalence of HIV and sexual behaviors among the US transgender population: a systematic review and metaanalysis, 2006-2017. Am J Public Health 2019;109:e1-e8.

3. Clark H, Babu AS, Wiewel EW, et al. Diagnosed HIV infection in transgender adults and adolescents: results from the National HIV Surveillance System, 2009-2014. AIDS Behav 2017;21:2774-83.

4. Hightow-Weidman LB, Muessig KE, Bauermeister J, et al. Youth, technology, and HIV: Recent advances and future directions. Curr HIV/AIDS Rep 2015;12:500-15.

5. Reback CJ, Rünger D. Technology use to facilitate health care among young adult transgender women living with HIV. AIDS Care 2020;32:785-92.

6. Stephenson R, Metheny N, Sharma A, et al. Providing home-based HIV testing and counseling for transgender youth (Project Moxie): Protocol for a pilot randomized controlled trial. JMIR Res Protoc 2017;6:e237.

7. Vance SR, Jr. The importance of getting the name right for transgender and other gender expansive youth. J Adolesc Health 2018;63:379-80.

8. Human Rights Campaign. 2018 gender-expansive youth report. Washington, DC, 2019. Available online: https://www.hrc.org/resources/2018-gender-expansiveyouth-report

9. Olson-Kennedy J, Cohen-Kettenis PT, Kreukels BPC, et al. Research priorities for gender nonconforming/ transgender youth: Gender identity development and biopsychosocial outcomes. Curr Opin Endocrinol Diabetes Obes 2016;23:172-9.

10. American Psychological Association. Guidelines for psychological practice with transgender and gender nonconforming people. Am Psychol 2015;70:832-64.

11. Schilt K, Westbrook L. Doing gender, doing heteronormativity: "Gender normals", transgender people, and the social maintenance of heterosexuality. Gend Soc
2009;23:440-64.

12. Graham R, Berkowitz B, Blum R, et al. The health of lesbian, gay, bisexual, and transgender people: Building a foundation for better understanding. Washington, DC: Institute of Medicine, 2011;10:13128.

13. Ehrensaft D. Exploring gender expansive expressions versus asserting a gender identity. In Keo-Meier C, Ehrensaft D, eds. The Gender affirmative model: An interdisciplinary approach to supporting transgender and gender expansive children. Washington, DC: American Psychological Association, 2018:37-53.

14. Nicholas L. Queer ethics and fostering positive mindsets toward nonbinary gender, genderqueer, and gender ambiguity. Int J Transgend 2018;20:169-80.

15. Herman JL, Flores AR, Brown TNT, et al. Age of individuals who identify as transgender in the United States. Los Angeles, CA, 2017.

16. Wilson BDM, Kastanis AA. Sexual and gender minority disproportionality and disparities in child welfare: A population-based study. Child Youth Serv Rev 2015;58:11-7.

17. Brennan J, Kuhns LM, Johnson AK, et al. Syndemic theory and HIV-related risk among young transgender women: The role of multiple, co-occurring health problems and social marginalization. Am J Public Health 2012;102:1751-7.

18. Wilson EC, Chen YH, Arayasirikul S, et al. Differential HIV risk for racial/ethnic minority trans* female youths and socioeconomic disparities in housing, residential stability, and education. Am J Public Health 2015;105:e41-7.

19. Garofalo R, Deleon J, Osmer E, et al. Overlooked, misunderstood and at-risk: Exploring the lives and HIV risk of ethnic minority male-to-female transgender youth. J Adolesc Health 2006;38:230-6.

20. James SE, Herman JL, Rankin S, et al. The report of the 2015 U.S. transgender survey. Washington, DC: National Center for Transgender Equality, 2016.

21. White Hughto JM, Reisner SL, Pachankis JE. Transgender stigma and health: A critical review of stigma determinants, mechanisms, and interventions. Soc Sci Med 2015;147:222-31.

22. Sevelius JM, Deutsch MB, Grant R. The future of PrEP among transgender women: The critical role of gender affirmation in research and clinical practices. J Int AIDS Soc 2016;19:21105.

23. Reisner SL, Radix A, Deutsch MB. Integrated and genderaffirming transgender clinical care and research. J Acquir 
Immune Defic Syndr 2016;72:S235-42.

24. Sevelius JM. Gender affirmation: A framework for conceptualizing risk behavior among transgender women of color. Sex Roles 2013;68:675-89.

25. Reisner SL, Bradford J, Hopwood R, et al. Comprehensive transgender healthcare: The gender affirming clinical and public health model of Fenway Health. J Urban Health 2015;92:584-92.

26. Turban JL, King D, Carswell JM, et al. Pubertal suppression for transgender youth and risk of suicidal ideation. Pediatrics 2020;145:e20191725.

27. Reisner SL, Jadwin-Cakmak L, Hughto JMW, et al. Characterizing the HIV prevention and care continua in a sample of transgender youth in the US. AIDS Behav 2017;21:3312-27.

28. Herbst JH, Jacobs ED, Finlayson TJ, et al. Estimating $\mathrm{HIV}$ prevalence and risk behaviors of transgender persons in the United States: a systematic review. AIDS Behav 2008;12:1-17.

29. Gay, Lesbian, \& Straight Education Network (GLSEN). Out online: The experiences of lesbian, gay, bisexual and transgender youth on the internet. New York, NY, 2013. Available online: https://www.glsen.org/news/out-onlineexperiences-lgbt-youth-internet

30. The Trevor Project. National survey on LGBTQ mental health. New York, NY, 2019. Available online: https:// www.thetrevorproject.org/survey-2019/

31. Adkins V, Masters E, Shumer D, et al. Exploring transgender adolescents' use of social media for support and health information seeking. J Adolesc Health 2018;62:S44.

32. boyd d. It's complicated: The social lives of networked teens. New Haven, CT: Yale University Press, 2014.

33. Carr CT, Hayes RA. Social media: Defining, developing, and divining. Atl J Commun 2015;23:46-65.

34. Cavalcante A. "I did it all online:" Transgender identity and the management of everyday life. Crit Stud Media Commun 2016;33:109-22.

35. Jackson SJ, Bailey M, Foucault Welles B. \#GirlsLikeUs: Trans advocacy and community building online. New Media Soc 2017;20:1868-88.

36. Jenzen O. Trans youth and social media: Moving between counterpublics and the wider web. Gend Place Cult 2017;24:1626-41.

37. Hawkins BW, Haimson O. Building an online community of care. Proceedings of the 4th Conference on Gender \& IT: GenderIT'18, 2018:75-7.

38. McDermott E, Roen K. Trans* and genderqueer youth online. In McDermott E, Roen K, eds. Queer youth, suicide, and self-harm: Troubled subjects, troubling norms. New York, NY: Palgrave Macmillan, 2016:80-102.

39. Evans YN, Gridley SJ, Crouch J, et al. Understanding online resource use by transgender youth and caregivers: A qualitative study. Transgend Health 2017;2:129-39.

40. Abreu RL, Kenny MC. Cyberbullying and LGBTQ youth: A systematic literature review and recommendations for prevention and intervention. J Child Adolesc Trauma 2017;11:81-97.

41. Cooper RM, Blumenfeld WJ. Responses to cyberbullying: A descriptive analysis of the frequency of and impact on LGBT and allied youth. J LGBT Youth 2012;9:153-77.

42. Scheuerman MK, Branham SM, Hamidi F. Safe spaces and safe places: Unpacking technology-mediated experiences of safety and harm with transgender people. Available online: https://dl.acm.org/doi/pdf/10.1145/3274424

43. Reback CJ, Horvath KJ, Rusow JA, et al. Technologybased stepped care to stem transgender adolescent risk transmission: Study protocol for a randomized controlled trial (TechStep). Available online: https://clinicaltrials.gov/ ct2/show/NCT04000724

44. Martin S. Developing and validating QueerViBE: An online intervention to empower trans and nonbinary youth. Cambridge, UK: Anglia Ruskin University, 2019.

45. Starks DL, Dillahunt T, Haimson OL. Designing technology to support safety for transgender women \& nonbinary people of color. Companion Publication of the 2019 on Designing Interactive Systems Conference 2019: DIS '19:289-94.

46. Kearns L. Binder Reminder (computer program). Version 1.0.10. 2019.

47. Widmer T. Refuge Restrooms. 2019. Available online: http://www.refugerestrooms.org/

48. Anthony RK. Solace (computer program). Version 1.4. 2020.

49. Perez K. EVA: Exceptional Voice App (computer program). Version 2.3.5. 2019.

50. Nagy P, Neff G. Imagined affordance:

Reconstructing a keyword for communication theory. Available online: https://journals.sagepub.com/ doi/10.1177/2056305115603385

51. Leonardi PM. Theoretical foundations for the study of sociomateriality. Inform Org 2013;23:59-76.

52. Arksey H, O'Malley L. Scoping studies: Towards a methodological framework. Int J Soc Res Methodol 2005;8:19-32.

53. Levac D, Colquhan H, O'Brien KK. Scoping studies: Advancing the methodology. Implement Sci 2010;5:69. 
54. Holeman I, Cookson TP, Pagliari C. Digital technology for health sector governance in low and middle income countries: A scoping review. J Glob Health 2016;6:020408.

55. Lalloo C, Shah U, Birnie KA, et al. Commercially available smartphone apps to support postoperative pain self-management: Scoping review. JMIR mHealth uHealth 2017;5:e162.

56. Portz JD, Elsbernd K, Plys E, et al. Elements of social convoy theory in mobile health for palliative care: Scoping review. JMIR mHealth uHealth 2020;8:e16060.

57. Rivera J, McPherson A, Hamilton J, et al. Mobile apps for weight management: A scoping review. JMIR mHealth uHealth 2016;4:e87.

58. Adepoju I-OO,Alberson BJA, De Brouwere V, et al. mHealth for clinical decision-making in Sub-Saharan Africa: A scoping review. JMIR mHealth uHealth 2017;5:e38.

59. IBM. Custom software development. Available online: https://www.ibm.com/topics/custom-softwaredevelopment

60. IBM. Mobile technology. Available online: https://www. ibm.com/topics/mobile-technology

61. World Health Organization. mHealth: New horizons for health through mobile technologies. Geneva, Switzerland, 2011. Available online: https://www.who.int/goe/ publications/goe_mhealth_web.pdf

62. Rafferty J, AAP Committee on Psychosocial Aspects of Child and Family Health, AAP Committeee on Adolescence, et al. Ensuring comprehensive care and support for transgender and gender-diverse children and adolescents. Pediatrics 2018;142:e20182162.

63. DuckDuckGo Blog. Measuring the "filter bubble": How google is influencing what you click. 2-18 Dec 4. Available online: https://spreadprivacy.com/google-filterbubble-study/

64. Zotero (computer program). Version 5.0.85. Available online: http://zotero.org

65. Hightow-Weidman LB, Muessig K, Rosenberg E, et al. University of North Carolina/Emory Center for Innovative Technology (iTech) for addressing the HIV epidemic among adolescents and young adults in the United States: Protocol and rationale for center development. JMIR Res Protoc 2018;7:e10365

66. Hong YR, Lawrence J, Williams D, et al. Population-level interest and telehealth capacity of US hospitals in response to COVID-19: Cross-sectional analysis of Google search and National Hospital Survey data. JMIR Public Health Surveill 2020;6:e18961.
67. Wosik J, Fudim M, Cameron B, et al. Telehealth transformation: COVID-19 and the rise of virtual care. J Am Med Inform Assoc 2020;27:957-62.

68. Peitzmeier S, Gardner I, Weinand J, et al. Health impact of chest binding among transgender adults: A community-engaged, cross-sectional study. Cult Health Sex 2017;19:64-75.

69. Speechtools Ltd. Christella VoiceUp (computer program). Version 3.0. 2020.

70. Ahmed AA. Bridging social critique and design. Extended Abstracts of the 2019 CHI Conference on Human Factors in Computing Systems: CHI EA'19, 2019:1-4.

71. Ahmed AA. Trans competent interaction design: A qualitative study on voice, identity, and technology. Interact Comput 2018;30:53-71.

72. Smith J. PatchDay (computer program). Version 2.2. 2019.

73. Stephenson R, Todd, K, Kahle, E, et al. Project Moxie: Results of a feasibility study of a telehealth intervention to increase HIV testing among binary and nonbinary transgender youth. AIDS Behav 2020;24:1517-30.

74. Naar S, Safren S. Motivational interviewing and CBT: Combining strategies for maximum effectiveness. New York: Guilford Press, 2017.

75. QueerDoc. Available online: https://queerdoc.com/

76. QMed. QMed: Providing affirming care for transgender \& nonbinary kids, teens \& adults. Decatur, GA, 2018. Available online: https://www.queermed.com/

77. Miller WK. Trust and antitrust: State-based restrictions in telemedicine. UC Davis L Rev, 2017;50:1807-44.

78. Connell RW. Masculinities. Cambridge: Polity Press, 1995.

79. Butler J. Gender trouble and the subversion of identity. London: Routledge, 1990.

80. Nadal KL. Microaggressions and traumatic stress: Theory, research, and clinical treatment. Washington, DC: American Psychological Association, 2018.

81. Addis ME, Reigeluth CS, Schwab JR. Social norms, social construction, and the psychology of men and masculinity. In: YJ Wong, Wester R, eds. APA handbook of men and masculinities. Washington, DC: American Psychological Association, 2016:81-104.

82. Foucault $M$. The history of sexuality: Volume 1 - An introduction. New York, NY: Vintage, 1978.

83. Foucault M. Power/knowledge: Selected interviews and other writings 1972-1977. Hassocks, Sussex: Harvester, 1980.

84. Beirl D, Zeitlin A, Chan J, et al. GotYourBack: An internet of toilets for the trans* community. Proceedings of the 2017 CHI Conference Extended Abstracts on Human Factors in Computing Systems: CHI EA '17, 2017:39-45. 
85. National Center for Transgender Equality. New iphone app finds nearby gender neutral restrooms. Washington, DC, 2012. Available online: https://transequality.org/blog/ new-iphone-app-finds-nearby-gender-neutral-restrooms

86. Kaufman K. Safe Transgender Bathroom App (computer program). Version 1.3. 2020.

87. Scholl J. YoRestrooms. Available online: https://yorestrooms.herokuapp.com/

88. Kirley A. ShotTraX (computer program). Version 1.5.4. 2020.

89. Wilson C. TransTracks (computer program). Version 1.1.0. 2019.

90. Anthony RK. Spokane Trans Map (computer program). Version 1.0.6. 2019.

91. The information-motivation-behavioral skills model: A general social psychological approach to understanding and promoting health behavior. In: Suls J, Wallston KA, editors. Social psychological foundations of health and illness. Malden: Blackwell Publishing, 2003:82-106.

92. Janz NK, Becker MH. The health belief model: A decade later. Health Educ Q 1984;11:1-47.

93. Bandura A. Social cognitive theory of self-regulation. Organ Behav Hum Decis Process 1991;50:248-87.

94. Turner RJ, Turner JB. Handbook of the sociology of mental health. New York: Springer, 1999.

95. Veiel HO, Baumann U. The meaning and measurement of support. London: Taylor \& Francis.

96. Reback CJ, Fletcher JB, Fehrenbacher AE, et al. Text messaging to improve linkage, retention, and health outcomes among HIV-positive young transgender women: Protocol for a randomized controlled trial (Text Me, Girl!). JMIR Res Protoc 2019;8:e12837.

97. Reback C. Text messaging to improve linkage, retention and health outcomes among HIV-positive young transgender women: Text Me Girl! Demonstration site summary. 2019. Available online: https://targethiv.org/ sites/default/files/supporting-files/spns-smi-text-me-girlmanual-508.pdf

98. Y'TH: Youth Tech Health. they2ze (computer program). Version 1.1. 2017.

99. Chrysalide. Trans Memo (computer program). Version 4.1.4. 2019.

100. Garcia M. Design and early clinical experience with a smartphone-based biofeedback app to improve pelvic floor muscle training (Kegel exercises) and routine neovagina self-care after transgender gender affirming surgery. J Sex Med 2017;14:e92-e3.

101.Sun CJ, Anderson KM, Mayer L, et al. Findings from formative research to develop a strength-based HIV prevention and sexual health promotion mHealth intervention for transgender women. Transgender Health 2019;4:350-8.

102.Sun CJ, Anderson KM, Kuhn T, et al. Development, usability testing, and acceptability of an mHealth HIV prevention intervention for transgender women: The Trans Women Connected mobile app. JMIR Preprints 2019. Available online: https://preprints.jmir.org/ preprint/15888

103. Magnus M, Edwards E, Dright A, et al. Development of a telehealth intervention to promote care-seeking among transgender women of color in Washington, DC. Public Health Nurs 2020;37:262-71.

104. Magnus M, Edwards E, Dright A, et al. A feasibility study of a telehealth intervention on health care service utilization among transgender women of color. Washington, DC: ACI Open 2018;2:e1-9.

105. Bothwell LE, Greene JA, Podolsky SH, et al. Assessing the gold standard - Lessons from the history of RCTs. N Engl J Med 2016;374:2175-81.

106. Sambasivan N, Checkley G, Ahmed N, et al. Gender equity in technologies. ACM Interactions 2017;25:58-61.

107. Stotzer RL. Law enforcement and criminal justice personnel interactions with transgender people in the United States: A literature review. Aggress Behav 2014;19:263-77.

108. Marcu G, Dowshen N, Saha S, et al. TreatYoSelf: Empathy-driven behavioral intervention for marginalized youth living with HIV. PervasiveHealth '16: Proceedings of the 10th EAI International Conference on Pervasive Computing Technologies for Healthcare 2016:69-76.

109. Haimson OL, Dykee G, Starks DL, et al. Designing trans technology: Defining challenges and envisioning community-centered solutions. ACM CHI Conference on Human Factors in Computing Systems 2020.

110. Elliott B. Anything is possible: Managing feature creep in an innovation rich environment. 2007 IEEE International Engineering Management Conference, 2007:304-7.

doi: $10.21037 /$ mhealth-20-60

Cite this article as: Skeen SJ, Cain D, Gamarel KE, HightowWeidman L, Reback CJ. mHealth for transgender and gender-expansive youth: harnessing gender-affirmative crossdisciplinary innovations to advance HIV prevention and care interventions. mHealth 2021;7:37. 